\title{
Colangiocarcinoma intrahépatico en hembra de la raza Springer Spaniel
}

\author{
Gennyffer M. Rincón, Est., Javier H. Albarracín-Navas, MVZ, Luz Z. Duarte-Rodríguez ${ }^{\star}, \mathrm{MVZ}_{1}$
}

Recibido: 25 de agosto del 2014. Aprobado: 4 de noviembre del 2014.

*Autor de correspondencia: Luz Zoraya Duarte R. Universidad Cooperativa de Colombia, Bucaramanga, Colombia. Calle 30A \# 33-51. Teléfono: (57) 7 6854500, ext. 7072. Correo electrónico: luz.duarte@campusucc.edu.co

Cómo citar este artículo: Rincón GM, Albarracín-Navas JH, Duarte-Rodríguez LZ. Colangiocarcinoma intrahépatico en hembra de la raza Springer Spaniel. Spei Domus. 2014;10(20):49-56. doi: http://dx.doi.org/10.16925/sp.v10i21.921

Resumen. El colangiocarcinoma o CGC es un tumor primario intrahépatico que se caracteriza por generar clínicamente signos inespecíficos de difícil aproximación diagnóstica; la citología dirigida con ecografía o laparotomía exploratoria facilitan su análisis, pero con el agravante de que el crecimiento tumoral en la mayoría de los casos está comprometiendo en un $60 \%$ aproximadamente el tejido hepático. El diagnóstico histopatológico permite determinar el origen primario del tumor como herramienta para su identificación y pronóstico del curso de la enfermedad. Los hallazgos clínicos no indicaron insuficiencia hepática, pero sí se evidenció hipoproteinemia y ecográficamente irregularidad de la silueta hepática para el presente caso.

Palabras clave: carcinoma, conductos biliares, geronte, hepáticos, neoplasia.

Intrahepatic Cholangiocarcinoma in Bitches of the Springer Spaniel Breed

\begin{abstract}
Cholangiocarcinoma or CGC is a intrahepatic primary tumor characterized by its generating of nonspecific signs that are difficult to diagnose. Ultrasound-guided cytology or exploratory laparotomy help analyze it, with the drawback being that in most cases the growth of the tumor is compromising approximately $60 \%$ of the liver tissue. Histopathological diagnosis allows the identification and prognosis of the disease's progress. Clinical findings did not indicate liver failure, but in this case they did show hypoproteinemia and abnormalities in liver silhouette ultrasounds.
\end{abstract}

Keywords: carcinoma, bile ducts, aging, hepatic, neoplasia.

\section{Colangiocarcinoma intra-hepático na fêmea da raça Springer Spaniel}

Resumo. O Colangiocarcinoma ou CGC é um tumor primário intrahepático que se caracteriza por gerar clinicamente signos inespecíficos de difícil aproximação diagnóstica, a citologia dirigida com ecografia ou laparotomia exploratória facilita sua análise, mas com o agravante de que o crescimento tumoral na maioria dos casos está comprometendo em $60 \%$ aproximadamente o tecido hepático. O diagnóstico histopatológico permite determinar a origem primária do tumor como ferramentas para sua identificação e prognóstico do curso da doença. As descobertas clínicas não indicaram insuficiência hepática, mas sim se evidenciou hipoproteinemia e ecograficamente irregularidade da silueta hepática para o presente caso.

Palavras-chave: carcinoma, condutos biliares, geronte, hepáticos, neoplasia. 


\section{Introducción}

Neoplasia (del griego "neo", nuevo y "plasis", formación) es la proliferación de clones de células atípicas, sin causa aparente de crecimiento excesivo, progresivo e ilimitado, no coordinado y autónomo [1]. Las células modificadas son ventajosas para la proliferación y la resistencia a la inducción de mecanismos de muerte celular, que culmina en el mantenimiento de células genéticamente alteradas en el tejido de origen. Con el tiempo, las células modificadas pueden adquirir la capacidad de invasión local, la inducción de cambios sostenidos en el microambiente del tejido, tales como la formación de nuevos vasos sanguíneos (angiogénesis) y, finalmente, la capacidad metastásica [2].

"El hígado es un órgano de frecuente aparición de neoplasias, pueden ser malignas y benignas; las neoplasias hepáticas primarias se clasifican según el origen embriológico en epiteliales y mesenquimales; adicionalmente, en el hígado se manifiestan varias neoplasias metastásicas" [3]. La prevalencia de neoplasias primarias hepatobiliares tienen un rango desde $0,6 \%$ a $2,6 \%$ en estudios de necropsia en caninos $[4,5]$.

El colangiocarcinoma (CGC) o carcinoma de conductos biliares es una neoplasia hepática primaria rara; se reporta en animales domésticos, especialmente en caninos y felinos, pero poco en otras especies [3], con alta morbilidad y mortalidad [6]. También llamado carcinoma colangiocelular $[7,8]$, es un tumor maligno de las células epiteliales que tapizan el ducto biliar [9], afectando tanto los conductos biliares intrahepáticos como los extrahepáticos [8]. En caninos, la forma intrahépatica es más frecuente, al ser el segundo tumor maligno primario más común; representa el $22-44 \%$ de todos los tumores malignos del hígado en esta especie [10]. Ha sido reportado en gatos viejos, y en ellos es la neoplasia hepática más frecuente, no se conoce que tenga predilección por raza en esta especie [11]. No hay una predisposición racial en caninos, pero la raza Labrador parece estar más predispuesta [10]; afecta con frecuencia a perros mayores de diez años de edad y las hembras esterilizadas tienen mayor riesgo que las hembras enteras o machos [8].

Los principales factores etiológicos son de naturaleza exógena, incluyendo las radiaciones, agentes químicos y virus [12]. Las manifestaciones clínicas de las neoplasias hepatobiliares en felinos son inespecíficas, incluyendo letargia, vómito, pérdida de peso, disnea, polidipsia, poliurea, ascitis, hipoalbuminemia y hepatomegalia [11-14]. Igualmente, se reportan en caninos signos clínicos inespecíficos asociados a disfunción hepática, como hepatomegalia, ictericia, vómito, anorexia, distención abdominal, encefalopatía hepática, ascitis y pérdida de peso [8]. Al examen clínico, la hepatomegalia es un hallazgo común y los bordes del órgano son irregulares, también puede encontrarse dolor o presencia de masas a la palpación [3].

En forma masiva, el tumor puede establecerse en un lóbulo completo y extenderse a los lóbulos adyacentes, en la forma multinodular. El tamaño del tumor puede variar de 0,5 a $4 \mathrm{~cm}$ de diámetro y estar distribuido por todos los lóbulos [10].

Autores reportan que en 137 muestras con diagnóstico de neoplasia se describió compromiso hepático, correspondiendo a procesos metastásicos en el $95,6 \%$ de ellas [3]. Las condiciones primarias $(4,4 \%)$ tuvieron origen en los canalículos biliares intrahepáticos en gallinas, bovinos y caninos. Los perros fueron mestizos, de 10 y 15 años de edad, respectivamente [3]. Las metástasis son frecuentes a ganglios linfáticos hepáticos, pulmón y peritoneo [12].

Macroscópicamente, pueden tener apariencia masiva o multinodular, incluso pueden reemplazar lóbulos completos y diseminarse a los adyacentes [11]. En la superficie de corte, el color varía de blanco a amarillo-café, y la textura es firme por la cantidad de tejido conectivo, lo cual es un criterio para diferenciarlo macroscópicamente del carcinoma hepatocelular [3].

En los CGC bien diferenciados histopatológicamente, las células semejan el epitelio biliar, son arregladas en forma tubular o acinar; los menos diferenciados tienen forma acinar y los pobremente diferenciados son compuestos de paquetes, islas o cordones celulares en los que se pueden presentar áreas con diferenciación escamosa y una intensa desmoplasia, con un estroma denso de colágeno que separa los elementos glandulares. El pronóstico es pobre debido a su alto poder metastásico [11].

La radiografía y la ultrasonografía son otras ayudas paraclínicas que pueden usarse para el diagnóstico de neoplasias hepáticas, pero la sensibilidad es variable [3]. La inmunohistoquímica juega un papel crucial en el diagnóstico de carcinoma hepatocelular y para su diferenciación de otras neoplasias primarias y metastásicas [15]. 
El diagnóstico definitivo de neoplasia hepática es por biopsia, con su respectivo análisis histopatológico [3]; el diagnóstico diferencial del CGC debe hacerse con el carcinoma hepatocelular variante adenoide, colangioma y carcinoide hepático [3].

\section{Reporte de caso}

Ingresa a la Clínica Pequeños Animales, de la Facultad de Medicina Veterinaria y Zootecnia de la Universidad Cooperativa de Colombia, un paciente canino hembra de raza Springer spaniel de 11 años de edad, con un peso de $19 \mathrm{~kg}$, con condición corporal 3/5, con manifestaciones de secreción purulenta por la vagina y evidencia de aumento de la silueta abdominal.

Al paciente se le realizó examen clínico, presentó temperatura de $39,4{ }^{\circ} \mathrm{C}$, frecuencia cardiaca de 102 $1 / \mathrm{m}$, frecuencia respiratoria de $46 \mathrm{r} / \mathrm{m}$, con actitud alerta. Paraclínicos como hemograma y bioquímica sanguínea (tablas 1 y 2). Los resultados no mostraron variaciones significativas; con leve anemia, leucocitosis y trombocitopenia moderada, así como una disminución en la albumina sérica.

Tabla 1. Cuadro hemático [16]

\begin{tabular}{|c|c|c|c|c|}
\hline Analito & $\begin{array}{c}\text { Valor } \\
\text { menor / mayor * }\end{array}$ & Unidad de medida & Val exm. & \\
\hline Micro hematocrito & 37 & 48 & $\%$ & 35 \\
\hline Hemoglobina & 12,5 & 16,0 & gr\% & 11,6 \\
\hline Recuento de leucocitos & 8000 & 13000 & $\mathrm{xmm}^{3}$ & 13850 \\
\hline Neutrófilos segmentados & 60 & 70 & $\%$ & 81 \\
\hline Linfocitos & 28 & 30 & $\%$ & 13 \\
\hline Monocitos & 3 & 10 & $\%$ & 3 \\
\hline Cayados o bandas & 0 & 1 & $\%$ & 3 \\
\hline Recuento de plaquetas & 150 & 450 & $\mathrm{X} 1000 / \mathrm{mm}^{3}$ & 140 \\
\hline Morfología de glóbulos rojos & & $\begin{array}{c}\text { Células diana ++ } \\
\text { Hipocromía }++ \\
\text { Policromatofilos + }\end{array}$ & & \\
\hline
\end{tabular}

Fuente: elaboración propia

Tabla 2. Bioquímica sanguínea

\begin{tabular}{|l|c|c|c|c|}
\hline \multicolumn{1}{|c|}{ Analito } & $\begin{array}{c}\text { Valor } \\
\text { menor / mayor }\end{array}$ & Unidad de medida & Val exm. & \\
\hline gpt-alt & 0 & 50 & $\mathrm{u} / \mathrm{l}$ & 23 \\
\hline Got-ast & 0 & 50 & $\mathrm{u} / \mathrm{m} / \mathrm{dl}$ & 41 \\
\hline Bun & 5 & 25 & $\mathrm{mg} / \mathrm{dl}$ & 16 \\
\hline Creatinina & 0,5 & 1,6 & $\mathrm{~g} / \mathrm{L}$ & 1 \\
\hline Globulinas & 29 & 39 & $\mathrm{~g} / \mathrm{L}$ & 30 \\
\hline Albumina & 26 & 33 & $\mathrm{~g} / \mathrm{L}$ & 21 \\
\hline Proteínas totales & 55 & 75 & & 52 \\
\hline
\end{tabular}

Fuente: [16]

Se realizó una ecografía en la que se encontró, a nivel del epigastrio (figura 1), una ecogenicidad mixta con marcada irregularidad, compatible con un proceso neoplásico (figura 2), ubicada en los lóbulos hepáticos, así como una alteración difusa del órgano (figura 3). 


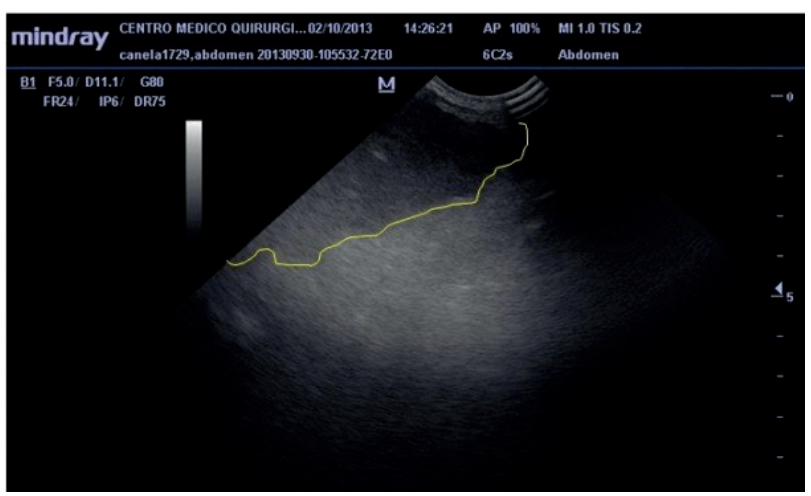

Figura 1. Ecografía abdominal del parénquima hepático Fuente: elaboración propia

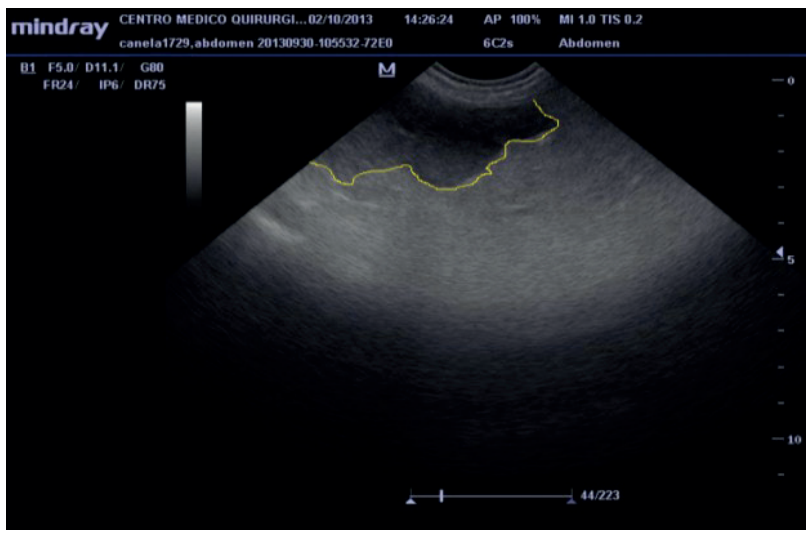

Figura 2. Ecografía abdominal, parénquima hepático con lesiones de distribución multifocal, compatible con masas tumorales Fuente: elaboración propia

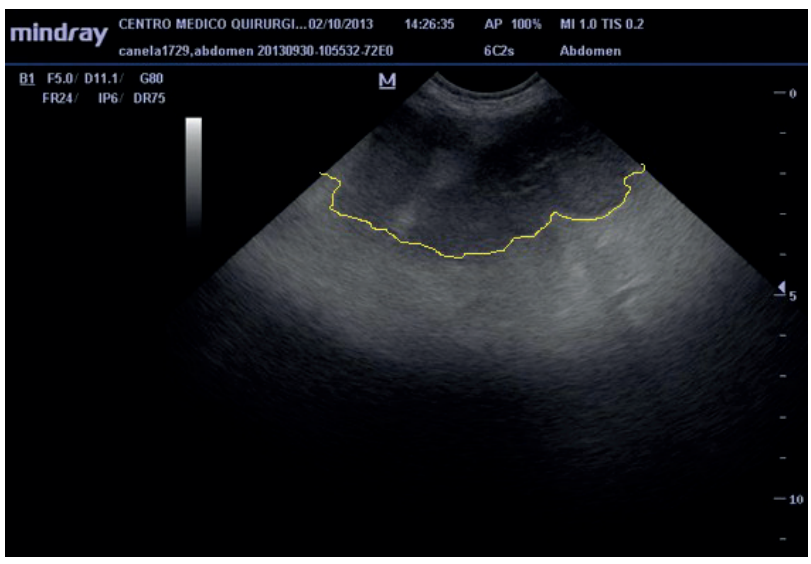

Figura 3. Ecografía abdominal, distribución difusa de áreas ecogénicas mixtas en lóbulo hepático Fuente: elaboración propia

En la radiografía simple de abdomen, en posición ventro dorsal (vD; figura 4), se observó aumento de la silueta hepática con unos bordes lobulares irregulares
(A) y desplazamiento hacia caudal de asas intestinales con contenido gaseoso (в), por lo cual se decidió realizar una RX con medio de contraste positivo.

También se tomó radiografía latero-lateral de abdomen con medio de contraste positivo (sulfato de Bario), en donde (A) indica desplazamiento hacia caudal de las asas intestinales por (B), el marcado aumento del tamaño de la silueta hepática (figura 5).

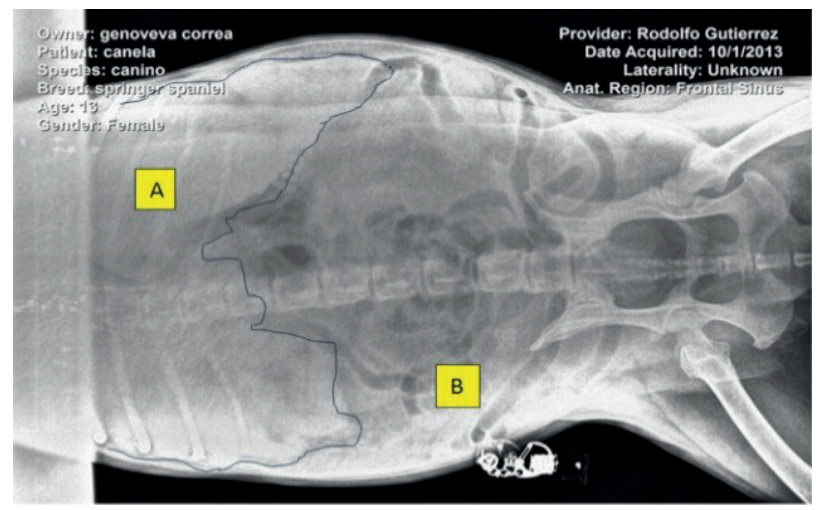

Figura 4. Radiografía vD. Hepatomegalia, bordes irregulares hepáticos

Fuente: elaboración propia.

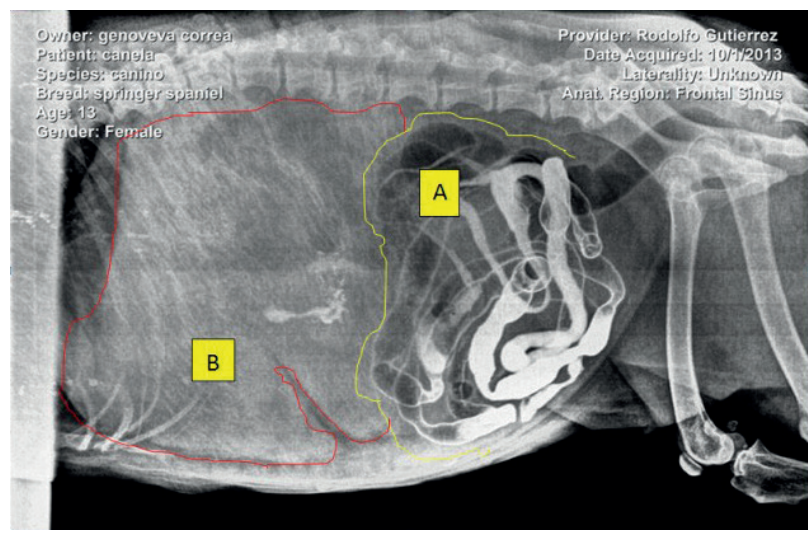

Figura 5. Radiografía abdominal, se evidencia hepatomegalia Fuente: elaboración propia

Dados los hallazgos ecográficos y radiográficos, se decidió practicar laparotomía exploratoria, confirmando los hallazgos radiográficos y ecográficos del parénquima hepático con aumento de peso del órgano y tamaño, con lesiones nodulares amarillentas de bordes elevados, con centro rojizo deprimido de distribución multifocal, afectando de manera multifocal el parénquima hepático (figuras $6,7,8$ ). 


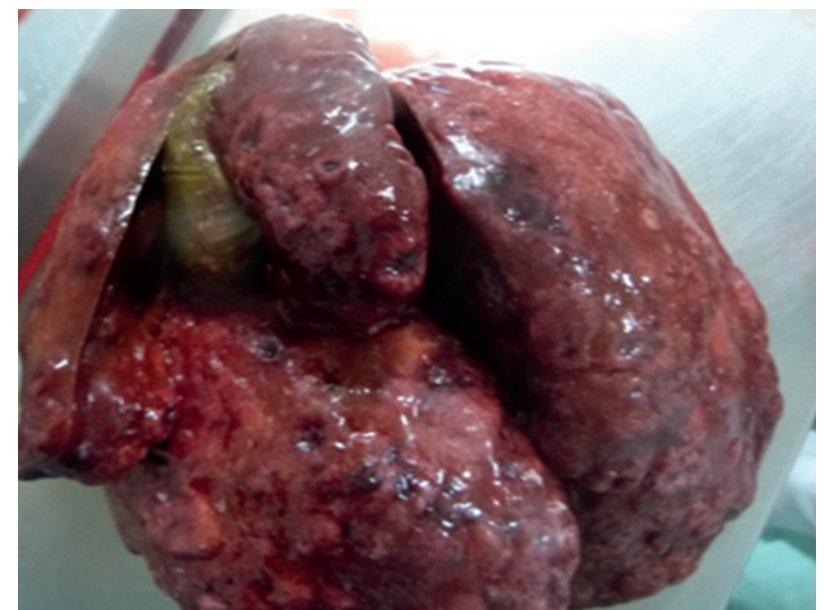

Figura 6. Lóbulos hepáticos irregulares con nódulos umbilicados afectando el parénquima hepático Fuente: elaboración propia

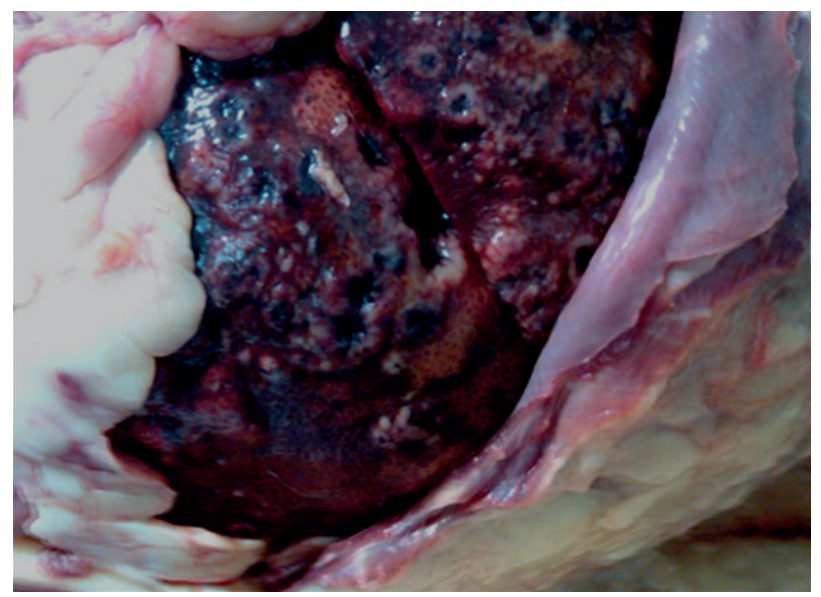

Figura 7. Lóbulos hepáticos irregulares con nódulos umbilicados afectando el parénquima hepático Fuente: elaboración propia

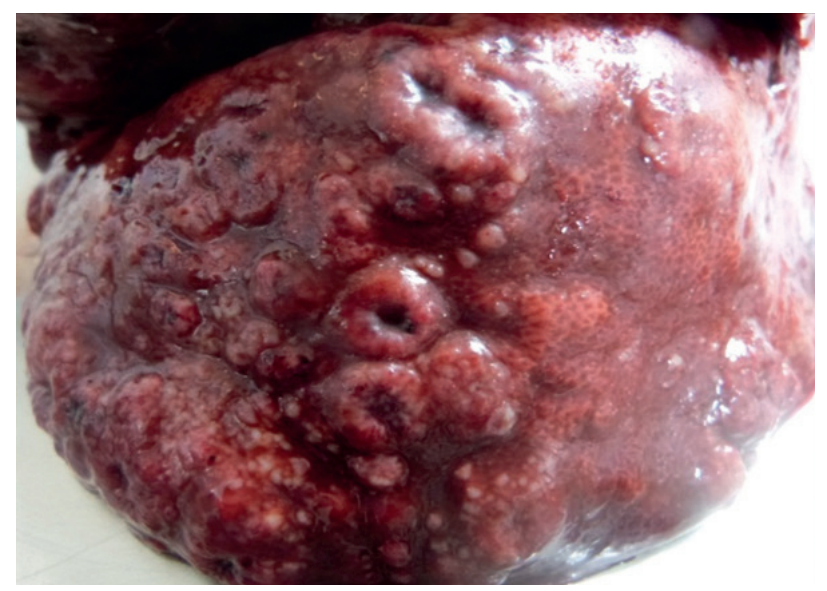

Figura 8. Detalle de los nódulos tumorales hepáticos Fuente: elaboración propia
Durante la exploración quirúrgica, se evidenció una segunda lesión nodular en el bazo (figura 9).

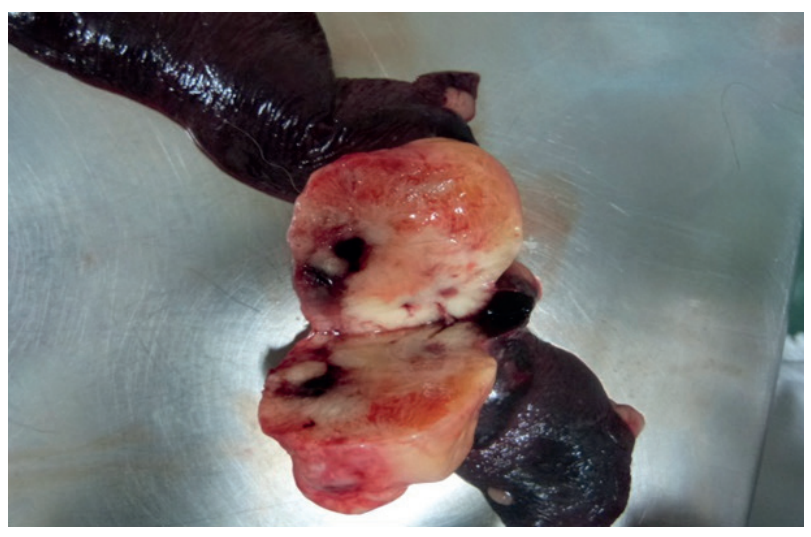

Figura 9. Nódulo esplénico de $3 \mathrm{~cm}$ aprox. de diámetro en el cuerpo del bazo

Fuente: elaboración propia

Una vez se evidenció el grado de infiltración del tumor y el pronóstico desfavorable del paciente, con autorización del propietario se decide realizar eutanasia y posterior necropsia del paciente, en las que se visualizó, además de lo detallado en las imágenes anteriores, ascitis, patrón nodular metastásico (figura 10) en la pared visceral abdominal, focos hemorrágicos en sistema digestivo, evidencia de edema traqueal, cistitis (figura 11) y ureteritis.

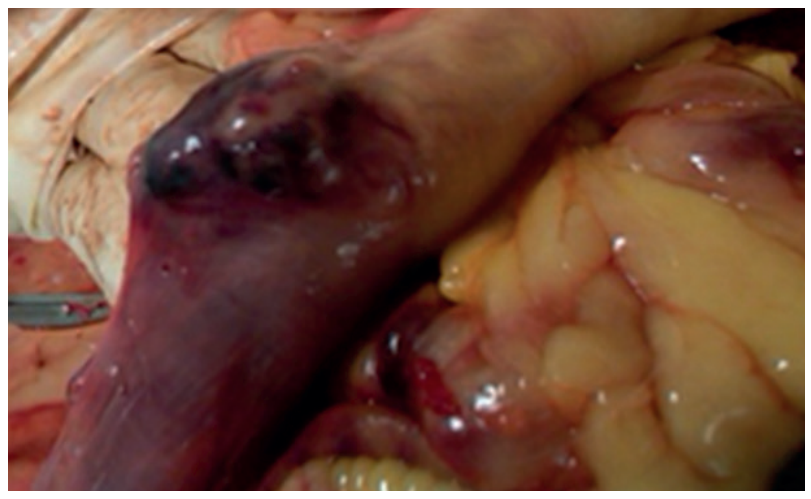

Figura 10. Nódulo metastásico en pared abdominal Fuente: elaboración propia 


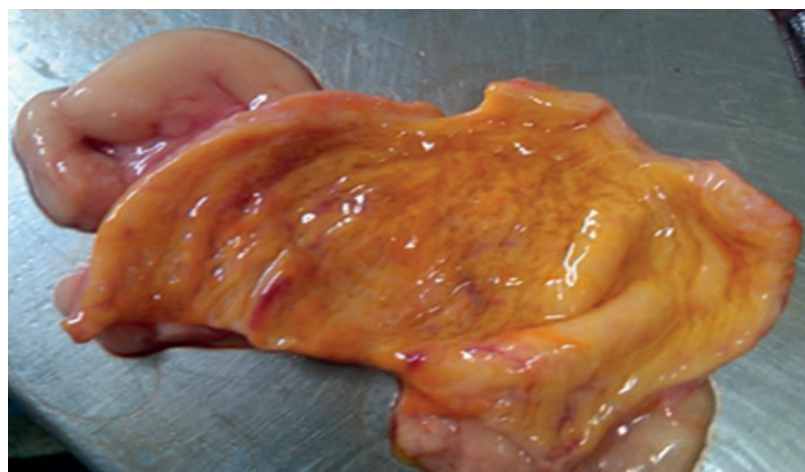

Figura 11. Cistitis nodular

Fuente: elaboración propia

\section{Morfo-histopatología}

En el tejido hepático, se observó que el comportamiento del crecimiento es infiltrativo (figura 12), el tumor se organiza en un patrón acinar sobre una matriz hialina de tejido conectivo que separa el crecimiento y le brinda soporte vascular (figura 13).

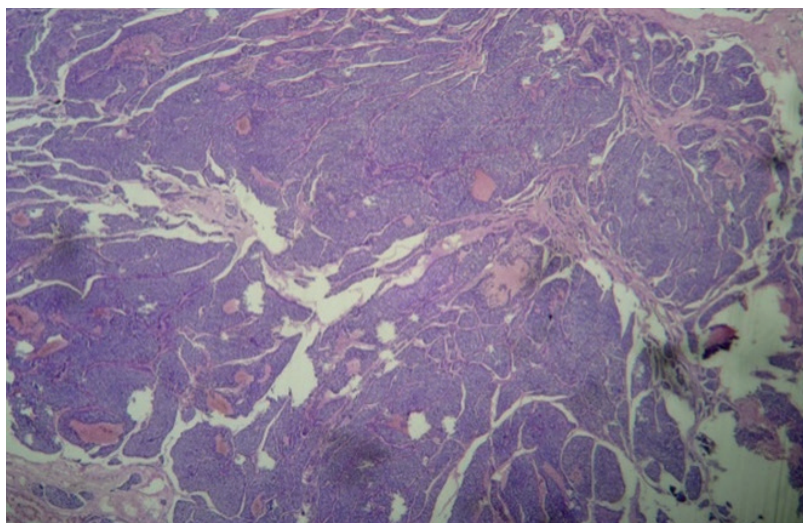

Figura 12. CGC hepático de crecimiento invasivo Fuente: elaboración propia

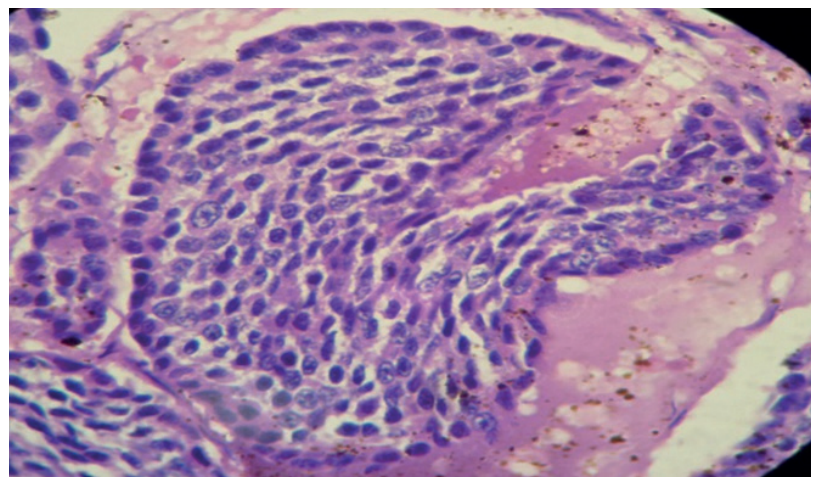

Figura 13. Morfología acinar de CGC intrahepático Fuente: elaboración propia
Los acinos presentan estratificación de células neoplásicas anaplásicas (figura 14). Las células periféricas tienen morfología cúbica a cilíndrica, indiferenciadas; la distribución central de las células indica pérdida de la polaridad celular, con núcleos hipercromáticos, cromatina vesicular, de citoplasma escaso eosinófilo y presencia de mitosis escasas.

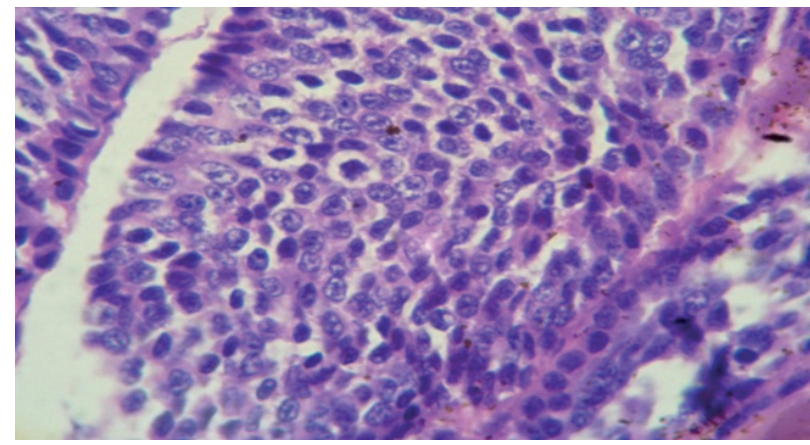

Figura 14. Proliferación celular anaplásica severa Fuente: elaboración propia

Se diagnostica para los hallazgos anatompatológicos colangiocarcinoma intrahépatico (CGC) tubular de patrón sólido.

\section{Discusión}

De acuerdo con lo reportado por la ecografía del paciente con el CGC, se observa que el tumor para este caso presentó ecogenicidad mixta con bordes hepáticos irregulares. Las neoplasias en hígado suelen ser hipoecogénicas o de ecogenicidad mixta, deformando el contorno cuando son de gran tamaño. En la necrosis central, característica de muchos tumores, se ve hipo o anecogénica y de contorno irregular [17].

En el cuadro hemático se evidenció trombocitopenia y leucocitosis moderada. Autores consultados explican que la leucocitosis suele estar asociada con una fuerte estimulación inmune, representada por la proliferación de células anormales (neoplásicas) [9]. La trombocitopenia es atribuida a una alteración en los procesos de coagulación, derivada del compromiso neoplásico esplénico o de la médula ósea por infiltración neoplásica o la producción de factores que inhiben la actividad trombopoyética por parte de las células neoplásicas [12], lo que puede estar asociado, para el caso, a la neoplasia primaria del fibrosarcoma esplénico reportado de manera incidental o a la inhibición 
de factores trombopoyéticos. De acuerdo con la bioquímica sanguínea, no se reportaron aumentos en los niveles de fosfatasa alcalina o de las aminotrasferasas que indicaran lesión hepática, pero coincide con otros autores en la hipoalbuminemia [3].

Las metástasis del CGC son frecuentes a ganglios linfáticos hepáticos regionales, pulmón y peritoneo [3, $8,18]$. Sin embargo, sólo se observó un patrón nodular localizado en la pared abdominal descrito macroscópicamente; no se evidenció distribución de este patrón multicéntrico en otros órganos.

En el bazo se encontró un nódulo irregular de color blanco grisáceo de aproximadamente $2,5 \mathrm{~cm}$ de diámetro, que histopatológicamente fue diagnosticado como fibrosarcoma esplénico, la descripción macro y microscópicamente es característica para el diagnóstico reportado por diversos autores [19].

El edema como hallazgo secundario del CGC está relacionado con la hipoproteinemia que se reportó [9, 20], asociado a la disfunción hepática por la disminución en la síntesis proteica.

Macroscópicamente, se evidenció en forma masiva la presencia de nódulos neoplásicos diseminados en los lóbulos hepáticos y se encontró una distribución igual a los reportes para el CGC [8], de consistencia firme, con aspecto umbilical, de coloración blanco a amarillo-rojizo variado, cuyas características son similares a otras descripciones reportadas $[8,10,18,20]$.

La mayoría de los CGC en caninos se presentan a la edad de diez años, con mayor riesgo en hembras castradas que en hembras enteras o machos $[3,18,20]$; esto coincide con factores de riesgo relacionados con el paciente, cuyas características con respecto al sexo y edad al ser hembra, castrada, mayor de diez años, indican una relación directa para la incidencia de la neoplasia.

Autores describen que el CGC moderadamente indiferenciado presenta intensa desmoplasia y un estroma denso de colágeno, que separa las estructuras glandulares, compuesto por células de epitelio biliar normal. En los CGC pobremente diferenciados se reporta que están compuestos por paquetes, islas o cordones, con focos adicionales de diferenciación escamosa [21-24]; para el presente caso, las características del CGC presentaron una distribución multifocal, cuya organización se da en islas de células estratificadas, con anaplasia celular $y$, al igual que el moderadamente indiferenciado, presenta un estroma denso de colágeno que divide el crecimiento.

\section{Conclusión}

En el caso se reporta el colangiocarcinoma intrahépatico CGC, como neoplasia primaria que explica la distensión abdominal por hepatomegalia e hipoproteinemia que generó desarrollo de ascitis [9], como lo indica la literatura; a pesar de la distribución multifocal de las lesiones nodulares neoplásicas. Estas se ubicaban en la superficie de corte sin compromiso del parénquima hepático, sin efectos sistémicos considerables o encefalopatía hepática secundaria. La edad está relacionada con la presentación del tumor, siendo los individuos adultos y gerontes los más propensos al diagnóstico con afección primaria neoplásica hepática al CGC.

Para el caso, no se presentó clínicamente la ictericia, vómito y la encefalopatía hepática $[11,18,20]$, pero sí la ascitis, distensión abdominal, hepatomegalia, con pérdida moderada de peso.

De manera secundaria, como hallazgo incidental, se observa en el tejido esplénico fibrosarcoma en el cuerpo del bazo, sin relación clínica importante, ni metastásica. El uso de ayudas diagnósticas inmediatas como el aspirado con aguja fina guiado por ecografía o sin ella, puede dilucidar inicialmente algunos diagnósticos de afección neoplásica hepática primaria [9], que son sugeridas para un diagnóstico en tumores primarios hepáticos.

\section{Referencias}

[1] Fossum TW. Cirurgia de pequenos animais. $1^{\mathrm{a}} \mathrm{ed}$. São Paulo: Roca; 2001.

[2] Chammas R, Silva D, Wainstein A, Abdallah K. Imunologia clinica das neoplasias. En: Imunologia Clínica na Prática Médica. São Paulo: Atheneu; 2009. p. 447-460.

[3] González G. Estudio retrospectivo de las neoplasias hepáticas en caninos en el laboratorio de patología veterinaria de la Universidad Nacional de Colombia entre los años 1975 y 2007 [tesis de grado]. [Bogotá]: Universidad Nacional de Colombia; 2010. Disponible en http://www.bdigital.unal.edu. co/2651/1/785027.2010.pdf

[4] Balkman C. Hepatobiliary Neoplasia in Dogs and Cats. Vet Clin Small Anim. 2009;39:617-25.

[5] Patnaik AK, Hurvitz AI, Lieberman PH. Canine Hepatic Neoplasms: A Clinicopathologic Study. Vet Pathology. 1980;17:553-64. 
[6] Javanbakht J, Sasani F, Khaki F, Jamshidi S, Hassan MA, Marzban H. Evaluation of Metastatic Cholangiocarcinoma in a Spitz Dog. J Cancer Sci The. 2013;5(3):113-4.

[7] Da Silva MC. Estudo retrospectivo de lesões hepáticas crônicas em cães [tesis de maestría]. [Santa María]:Universidade Federal deSanta Maria;2013. Disponible en: http://cascavel.cpd.ufsm.br/tede/ tde_arquivos/8/TDE-2007-07-05T085122Z-685/ Publico/MARCIA.pdf

[8] Boanova HA, Molarihno KR, Alves DM, Mainardi DM, Cioato da Silva C, Bonel J. Colangiocarcinoma em cão: relato de caso. Documento presentado en: XXII Congreso de Iniciação Científica da Universidade Federal de Pelotas. 2010 nov 18-22; Capão do Leão. Disponible en: http://cti.ufpel.edu. br/cic/arquivos/2013/CA_02697.pdf

[9] Buriticá E, Barbosa X, Echeverry D. Carcinoma hepatocelular canino: reporte de un caso. Rev Mvz Córdoba. 2009;14(2):1756-61. Disponible en: http://revistas.unicordoba.edu.co/revistamvz/ mvz-142/v14n2a12.pdf

[10] Teixeira L. Diagnóstico citológico de neoplasias hepatobiliares em cães atendidos no Hospital de Clínicas Veterinárias (HCV-UfRGs) no periodo de 2005 a 2010 [tesis de grado]. [Río Grande del Sur]: Universidade Federal do Rio Grande do Sul; 2011. Disponible en: http://www.lume.ufrgs. br/bitstream/handle/10183/38713/000794236. pdf? sequence $=1$

[11] Ochoa J, Roque AI, Daza JA. Colangiocarcinoma hepático en un felino y hallazgos anatomopatológicos y clínicos compatibles con peritonitis infecciosa. Rev mvz Córdoba. 2012;17(2):3080-6. Disponible en: http://revistas.unicordoba.edu.co/ revistamvz/mvz-172/V17N2A18.pdf

[12] Cruz RSI. Clasificación histológica según la Organización Mundial de la Salud y frecuencia de neoplasias en animales domésticos, aves y peces, durante el periodo 1967-1995 en Valdivia, Chile [trabajo de grado]. [Valdivia]: Universidad Austral de Chile; 1997. Disponible en: http://cybertesis.uach.cl/tesis/ uach/1997/fvi.27c/doc/fvi.27c.pdf

[13] Crawford JM. El hígado y las vías biliares. En: Cotran R, Kumar V, Collins T, editor. Robbins.
Patologia estructural y funcional. $6^{\mathrm{a}}$ ed. México: Interamericana; 2000.

[14] Cullen JM, Maclachlan. Liver, Biliary System and Exocrine Pancreas. En: McGavin DM, Carlton WW, Zachary JF. Thomson's Special Veterinary Pathology. Missouri: Mosby; 2001.

[15] Kakar S, Gown AM, Goodman ZD, Ferrell LD. Best Practices in Diagnostic Immunohistochemistry: Hepatocellular Carcinoma versus Metastatic Neoplasms. Arch Pathol Lab Med 2007;131(11):1648-54.

[16] Estupiñán A. Mercha C. Determinación de valores hematológicos y de química sanguínea en caninos clínicamente sanos en la ciudad de Bucaramanga [trabajo de grado]. [Bucaramanga]: Universidad Cooperativa de Colombia; 2008.

[17] Moreno A, Hervás J, Chacón, F. Significado patológico de la imagen ultrasónica en pequeños animales. Anales de la Real Academia de Ciencias Veterinarias de Andalucía Oriental. 2011;24:213-33.

[18] Pimentel MC, Fiss L, Franco M, Rossato CK. Colangiocarcinoma multinodular em cão: relato de dois casos. De Dois Casos. Documento presentado en: xv Seminário interinstitucional de ensino, pesquisa e extensão. 2010 nov 9-11; Cruz Alta.

[19] Toni P, Rollán E, Montoya JA, Rodríguez F. Fibrosarcoma esplénico primario en un perro. AVEPA. 1989;9(2):73-5.

[20] Silva FDAE. Hepatocarcinoma canino: relato de caso [internet]. 2008. Disponible en: https://www.equalis. com.br/arquivos_fck_editor/TCC FINNAL.pdf

[21] Watson P. wsava Standards for Clinical and Histological Diagnosis of Canine and Feline Liver Diseases. Filadelfia: Saunders Elsevier; 2007.

[22] Baba AI, Cornel C. Tumors of the Liver and Gallbladder. En: Comparative Oncology. Bucharest: The Publishing House of the Romanian Academy; 2007. Disponible en: http://www.ncbi.nlm.nih. gov/books/NBK9548/

[23] Nakanuma Y, Harada K, Ishikawa A, Zen Y, Sasaki M. Anatomic and Molecular Pathology of Intrahepatic Cholangiocarcinoma. J Hepatobiliary Pancreat Surg. 2003;10(4):265-81.

[24] Meuten DJ. Tumors in Domestic Animals, 4th ed. Review. Can Vet J. 2002;44(8):684. 